BBR 00393

\title{
DISTANCE ESTIMATION IN THE MONGOLIAN GERBIL: THE ROLE OF DYNAMIC DEPTH CUES
}

\author{
COLIN G. ELLARD, MELVYN A. GOODALE and BRIAN TIMNEY \\ Department of Psychology, University of Western Ontario, London, Ont. N6A 5 C2 (Canada)
}

(Received June 18th, 1984)

(Revised version received August 24th, 1984)

(Accepted September 4th, 1984)

Key words: depth vision - motion parallax - loom - head movement - jumping stand - gerbil

\begin{abstract}
The role of dynamic depth cues in distance estimation was investigated in the Mongolian gerbil. Animals were trained to jump randomly varied distances on a jumping stand under both binocular and monocular conditions. Videotape analysis revealed that prior to jumping, the gerbils executed a series of vertical head movements, the amplitude and velocity of which were related to the gap distance and to each animal's accuracy. This suggested that the gerbils were employing motion parallax cues to judge distance. An inverse relation between the magnitude of forward movement and the frequency of vertical head movements suggested that loom cues were also being used to judge distance. This hypothesis received support from a second experiment in which forward movements were constrained by a short take-off platform. In this condition, frequency of vertical head movements increased, suggesting that a compensation had occurred for the loss of information from loom.
\end{abstract}

\section{INTRODUCTION}

Dynamic cues to depth such as loom and motion parallax can provide a moving animal with precise information about the depth and distance of different surfaces in the world through which it is moving. While the utility of dynamic depth cues is well documented in $\operatorname{man}^{13,15}$, the use of such cues in lower animals has seldom been demonstrated although it is often assumed.

In a variety of experiments in which animals have been required to make depth judgements, a number of species have been shown to execute a series of oscillations of the head prior to the performance of the task ${ }^{1,2,10,16-18}$. While the suggestion has been made that the purpose of these head movements is to judge distance by generating motion parallax cues, this claim has been substantiated only in the locust ${ }^{2,18}$.
In a pilot study in which Mongolian gerbils had been trained to jump between two raised platforms, we noticed that they often executed a series of vertical translation movements of the head before jumping. The purpose of the present study was to determine whether these movements were being used to generate motion parallax cues. If so, one might predict that the dynamics of these movements should be systematically related to the task parameters (the size of the required jump, for instance) and to the animals's jumping performance. Measurements of the amplitude, velocity and frequency of these movements were extracted from videotapes made of the gerbils as they prepared to jump over the gap between the two platforms. These parameters could then be systematically related to the distance of the required jump and the accuracy of the gerbil's performance. 


\section{METHODS}

\section{Subjects}

Ten Mongolian gerbils (Meriones unguiculatus) approximately 90 days of age at the beginning of training were used. They were housed individually in a colony room on a $12: 12 \mathrm{~h}$ light-dark schedule. During training, animals were maintained at $80-85 \%$ of their free feeding weight. Once they had begun to perform well, they were maintained at $95-100 \%$ of their free feeding weight. Water was always freely available in the home cage.

\section{Apparatus}

The jumping apparatus consisted of two raised platforms $21 \mathrm{~cm}$ high and coated with coarse sandpaper to aid traction (Fig. 1). The 'take-off' platform included a rectangular pre-jump area $(20 \times 18 \mathrm{~cm})$ which was continuous with a short tapering take-off strip ( $8 \mathrm{~cm}$ long, $7.5 \mathrm{~cm}$ base width, $4 \mathrm{~cm}$ apical width). A rectangular landing platform $(30 \times 27 \mathrm{~cm})$ could be located at different distances from the take-off platform. A large background measuring $87 \times 60 \mathrm{~cm}$ and consisting of $1 \mathrm{~cm}$ grey and white squares organized in a random pattern was placed $71 \mathrm{~cm}$ behind the leading edge of the take-off platform. From this distance the squares subtended a visual angle of $0.81^{\circ}$, well within the acuity limits of the gerbil ${ }^{1}$.

Both platforms were uniformly illuminated from above by the reflected light from a photoflood lamp.

\section{Procedure}

Before any jumps were videotaped, each animal received at least 250 training trials on the jumping apparatus. Each animal was trained first to step across a small gap separating the take-off platform from the landing platform in order to obtain a shelled sunflower seed placed in the middle of the landing platform. Over successive trials, the gap distance was varied randomly but the mean size of the gap was gradually increased. Each animal received 25 of these training trials in a single session every 2 days until it had suecessfully jumped a distance of $35 \mathrm{~cm}$.

Following this training period, the 10 animals were each given 4 sessions of 26 test trials at distances ranging from 10 to $35 \mathrm{~cm}$ in $1 \mathrm{~cm}$ increments. Both the order of the distances required to

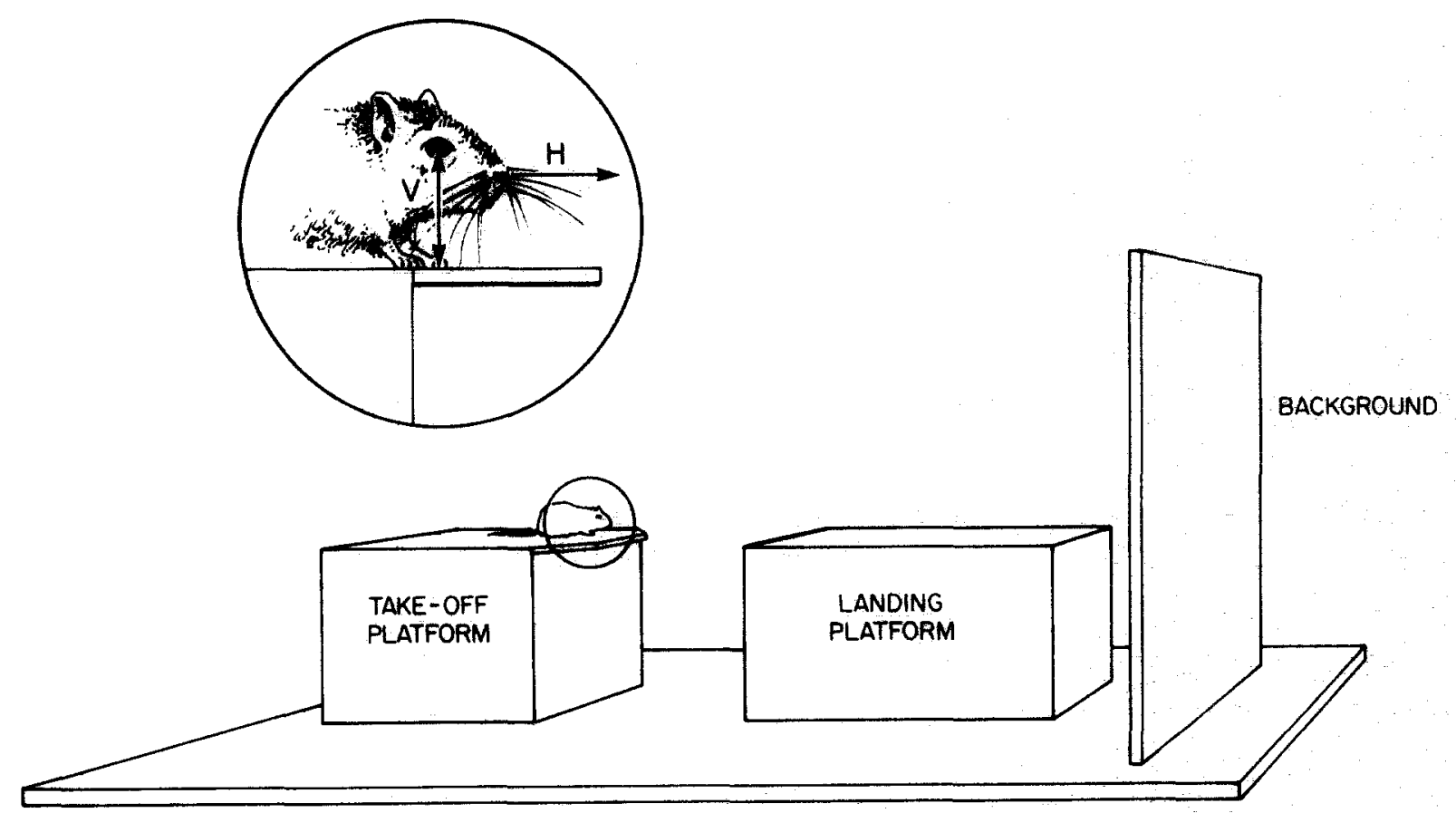

Fig. 1. A schematic diagram of the apparatus used to test visually guided jumping in the gerbil. The inset illustrates vertical (V) and horizontal $(\mathrm{H})$ displacements of the head. 
be jumped and the order of the animals tested at a given distance were randomized. A trial consisted of placing an animal on the back of the take-off platform facing away from the landing platform for a period of 1 min or until the animal concluded the trial by jumping from the take-off strip. Whether the animal succeeded in reaching the landing platform or not, every jump was scored as a trial and was treated in the same way in all subsequent analyses. These 4 sessions of test trials constituted Condition B 1, the first set of binocular test sessions.

After each animal had completed Condition B1, it was tested in 3 further sets of test sessions, two monocular (Conditions M1 and M2) and one binocular (Condition B2). Testing under each of these conditions again consisted of 4 sessions of 26 test trials.

Before monocular testing began, each animal had the lids of one eye sutured closed under deep sodium pentobarbital anesthesia $(50 \mathrm{mg} / \mathrm{kg})$. The eye was sutured closed without resecting the eye lids to permit easy reopening at the end of monocular testing. A generous application of antibiotic cream applied to the sutured eye at the start of each test session ensured that the eye was effectively occluded. After the two sets of monocular testing were completed (one session per day for 8 days), the closed eye was reopened under anesthesia and, following 7 days of recovery, the animals were tested in the final binocular test sessions (Condition B2).

The performance of each animal on these 4 sets of test sessions (Conditions B1, M1, M2 and B2) was recorded by means of two videocameras. The field of view of one camera, an Ikegami ITC-47, included the take-off strip and the front $3 \mathrm{~cm}$ of the pre-jump area. This camera therefore provided a magnified image of the animal's head prior to the jump. The field of view of the other camera, a Sony RSC-1050 fitted with a high-speed rotary shutter, included the take-off strip and at least one third of the landing platform. This camera was used to record the trajectory of the jump. The images from both cameras were synchronized and fed through a Tokina TW-104 splitter to a TEL video counter-timer which added a time display to the screen image with a resolution of $10 \mathrm{~ms}$. The combined image was then stored on a videocassette recorder.

In a final experiment, 6 of the gerbils were tested binocularly in a further condition in which the size of the take-off platform was reduced by requiring the animals to jump from a small $5 \times 5 \mathrm{~cm}$ platform attached to a retort stand. Each animal was videotaped as it jumped randomly ordered distances that ranged from 10 to $34 \mathrm{~cm}$ in $3 \mathrm{~cm}$ increments. Each animal received 4 trials at each distance tested.

\section{Analysis}

To quantify the movements of a gerbil both before and during a jump, each 'frame' of the videotape record was projected from a television monitor onto an $\mathrm{X}-\mathrm{Y}$ digitizing tablet via an opaque projection system ${ }^{11}$. The digitizing tablet was linked to an Apple II Plus microcomputer. By plotting the position of various body parts of the gerbil in Cartesian coordinates on successive frames of videotape (frame speed $=30$ frames $/ \mathrm{s}$ ), the following measurements were extracted from the videotape records of each trial:

(1) Head movements. A head movement was defined as the occurrence of at least two consecutive frames of upward or downward movement of the head in the period before the animal initiated a jump. The reference point for these movements was always the animal's eye (Fig. 1). Individual movements of the head were separated by pauses (where a pause was defined as at least two consecutive frames of immobility). For each head movement, the time of onset, duration, magnitude and velocity of the vertical displacement of the head was recorded.

(2) Leg movements. For each forward movement of the rear legs, the time of onset, duration, and magnitude of the horizontal displacement of the head was recorded. A leg movement was defined as beginning when one or both rear paws were lifted from the take-off platform and ending when both rear paws resumed contact with the platform. The reference point for the horizontal displacement was the animal's snout.

(3) Trajectory. The trajectory of the jump was also recorded. The beginning of the jump was defined as the last frame in which at least one 
forepaw was in contact with the take-off platform. The end of the jump was defined as the first frame in which at least one forepaw was in contact with the landing platform or, in the case of missed jumps, the first frame in which a forepaw reached the same height as the top of the landing platform. The trajectory of the jump was reconstructed from the position of the animal's snout on successive frames of videotape. The touchdown position of the forepaws on the landing platform was recorded for all correct jumps.

This analysis was performed on all trials for the first two sessions of Conditions B1, M1, M2 and B2 and on all trials of the last experiment with the small take-off platform.

\section{RESULTS}

All the gerbils learned to jump over the gap separating the two platforms with little difficulty. As Fig. 2 illustrates, the magnitude of their jump was strongly correlated with the size of the gap. Moreover, their jumps on test trials were quite accurate despite the fact that the size of the gap on any one trial was randomly determined.

The frequency of vertical head movements preceding the jump also varied as a function of the gap distance. The movements were divided into two categories: those with a vertical displacement smaller than $1.5 \mathrm{~cm}$, and those with a vertical displacement equal to or larger than $1.5 \mathrm{~cm}$. This value was chosen because it corresponds to the average interocular distance in the gerbil. Theoretically then, head movements that were smaller than this value would have provided less information about depth than that supplied by binocular disparity. The relationship between the frequency of these two classes of movement and the size of the gap is illustrated in Fig. 3. As this figure illustrates, the number of head bobs in both classes increased as a function of gap size in all 4 test conditions. It can be seen, however, that the relationship between the number of head bobs and the distance required to be jumped was far less pronounced in Condition M1 than in the other 3 conditions (see below). This appeared to be due largely to an increase in the number of head bobs (particularly those equal to or larger than
$1.5 \mathrm{~cm}$ ) at shorter gap distances in Condition M1. Thus, in contrast to Condition B1, where a total of only 28 large-amplitude head bobs were made by the 10 animals at gap distances of less than $23 \mathrm{~cm}$, in Condition $\mathrm{Ml}$, this number had risen more than 4-fold to 131. By Condition M2, the number of large-amplitude movements preceding jumps of less than $23 \mathrm{~cm}$ had fallen to 75 and by Condition B2 to only 23. This pattern of results is borne out by the correlations between gap distance and the proportion of head bobs that were equal to or larger than $1.5 \mathrm{~cm}$. While these correlations were highest in conditions B1, M2 and B2 $\left(r=0.65, t_{24}=4.19, r=0.50, t_{24}=2.83\right.$ and $r=0.73, t_{24}=5.23$, respectively, $P<0.01$, the correlation was lowest for condition M1 $\left(r=0.40, t_{24}=2.14, P<0.05\right)$. Since the amplitude and the peak velocity of the head movements were strongly correlated $\left(r=0.86, t_{3757}=103.3\right.$, $P<0.0001$ ), the proportion of high velocity movements of the head also increased as a function of the distance to be jumped.

At the end of a head movement, particularly a large one, a very small 'rebound' movement was often observed, perhaps indicating the action of antagonistic muscle groups during the deceleration phase of the movement. These rebound movements and the significant correlation obtained between peak velocity and amplitude of head movement suggested that the head movements were ballistic. To test this notion, the relationship between the initial acceleration phase and the peak velocity of the head movements was examined. Ballistic movements tend to have similar durations regardless of their amplitude and consequently exhibit a strong relationship between acceleration and peak velocity. The correlation between peak velocity and acceleration for the head bobs was $r=0.78 \quad\left(t_{3757}=76.4\right.$, $P<0.001$ ).

In all conditions, there was considerable variability in the number of head bobs produced by individual animals. Nevertheless, as Fig. 4 shows, the number of animals producing head bobs was strongly correlated with the distance they were required to jump. The measure used in this anal$y$ sis was the proportion of trials on which at least one head bob occurred. Again the performance of 

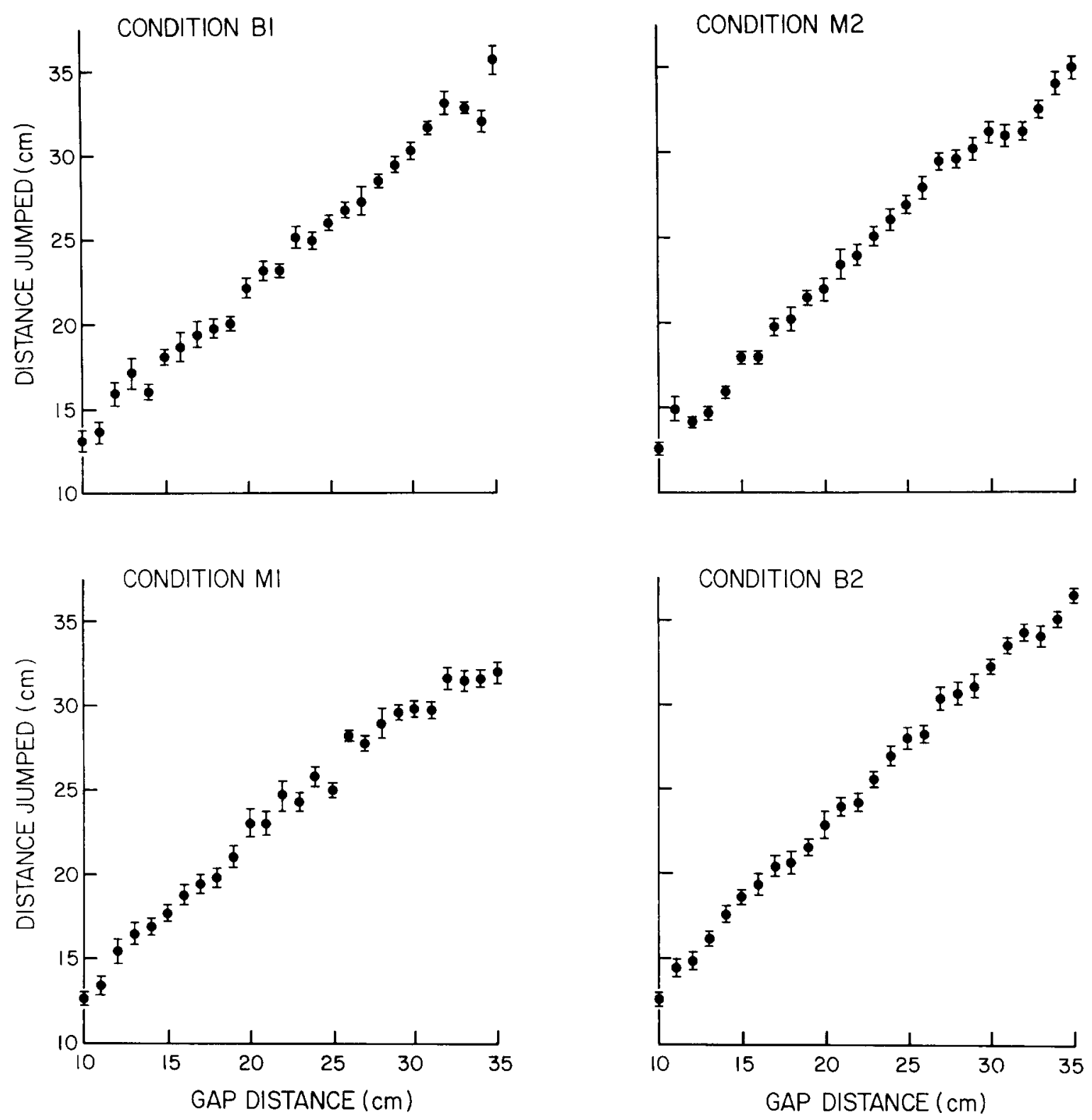

Fig. 2. Plots of the mean distance jumped as a function of gap distance. Data points represent the means over all animals and trials within each condition. Error bars represent the standard error of the mean. Each condition is plotted separately.

animals during Condition M1 was different from their performance in the other 3 conditions. During this first monocular condition, the proportion of animals producing head bobs was markedly raised, particularly at short distances. Indeed, when the ratios between these proportions in Conditions $\mathrm{M} 1$ and $\mathrm{B} 1$ were calculated for each gap distance (Fig. 5), there was a strong relationship between the size of this ratio and the distance required to be jumped $\left(r=-0.76, t_{24}=5.73\right.$,
$P<0.001$ ) suggesting that the production of head bobs during Condition M1 was selectively raised at short distances. Although the proportion of animals producing head bobs was also slightly higher during Condition M2 as compared to both binocular testing conditions, the same selective increase in the production of head bobs at short distances was not observed.

Although in most of the test sessions the number of head bobs increased as a function of the 

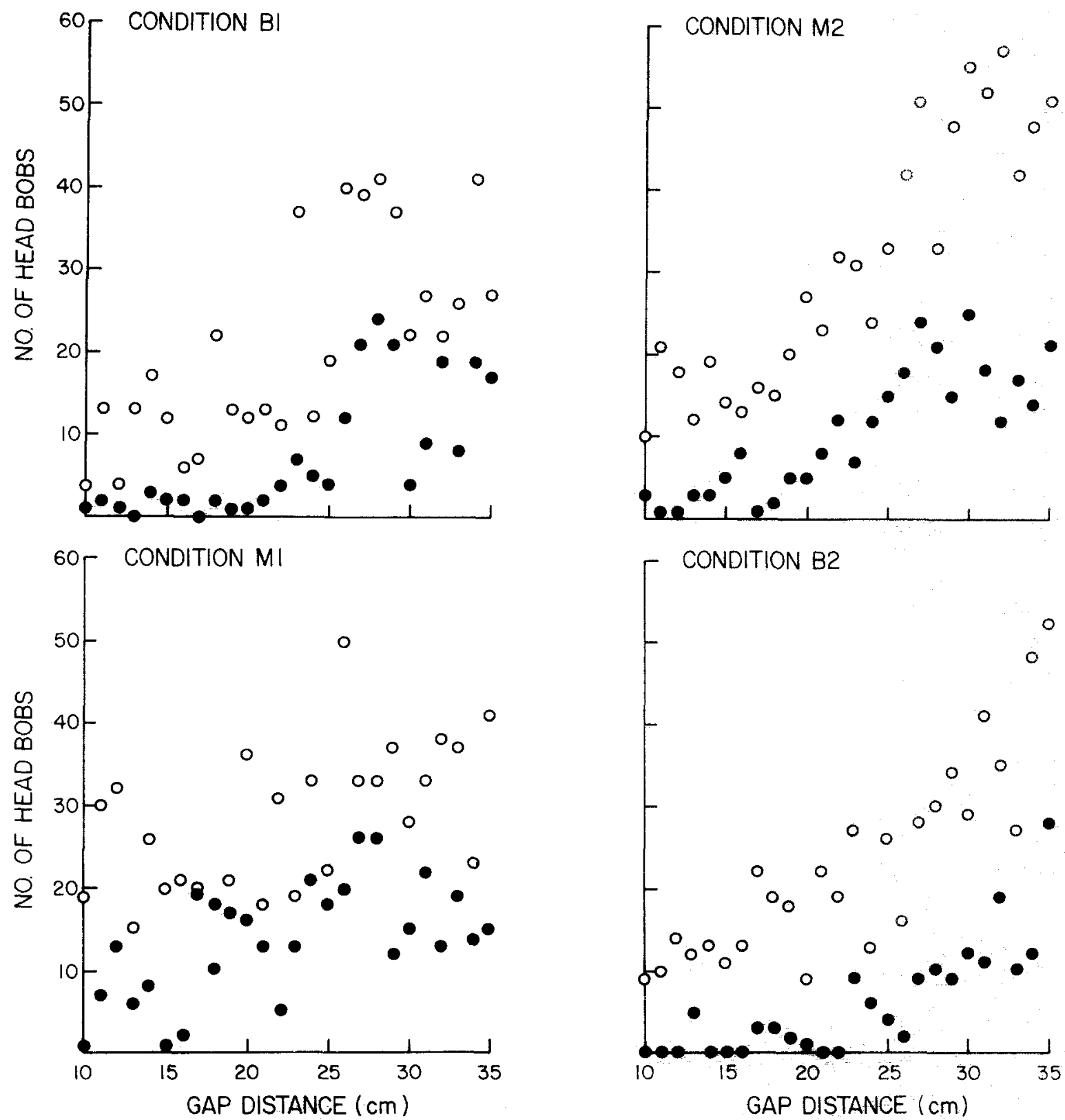

Fig. 3. Plots showing the frequency of head movements as a function of distance. Each data point represents the total number of head movements executed by all animals at that gap distance. Open circles represent head movements smaller than $1.5 \mathrm{~cm}$. Filled circles represent head movements larger than or equal to $1.5 \mathrm{~cm}$. Each experimental condition is plotted separately.

distance the gerbils were required to jump, the question remained as to the relationship between head bobs and the accuracy of the jump. Since the large landing platform did not place a significant demand for accuracy upon the animals, it was difficult to establish what an optimal jump should look like. In order to quantify the accuracy of an animal's performance, it was assumed that only those animals judging distance accurately would be consistent from trial to trial. Olson ${ }^{12}$ has shown that cats exhibit such trial-to-trial consistency in a jumping task. Thus, variance in landing position could be used as a measure of the accuracy of an animal's judgement of depth. It was then possible to relate this measure of accuracy to a measure of head bob incidence. Head 

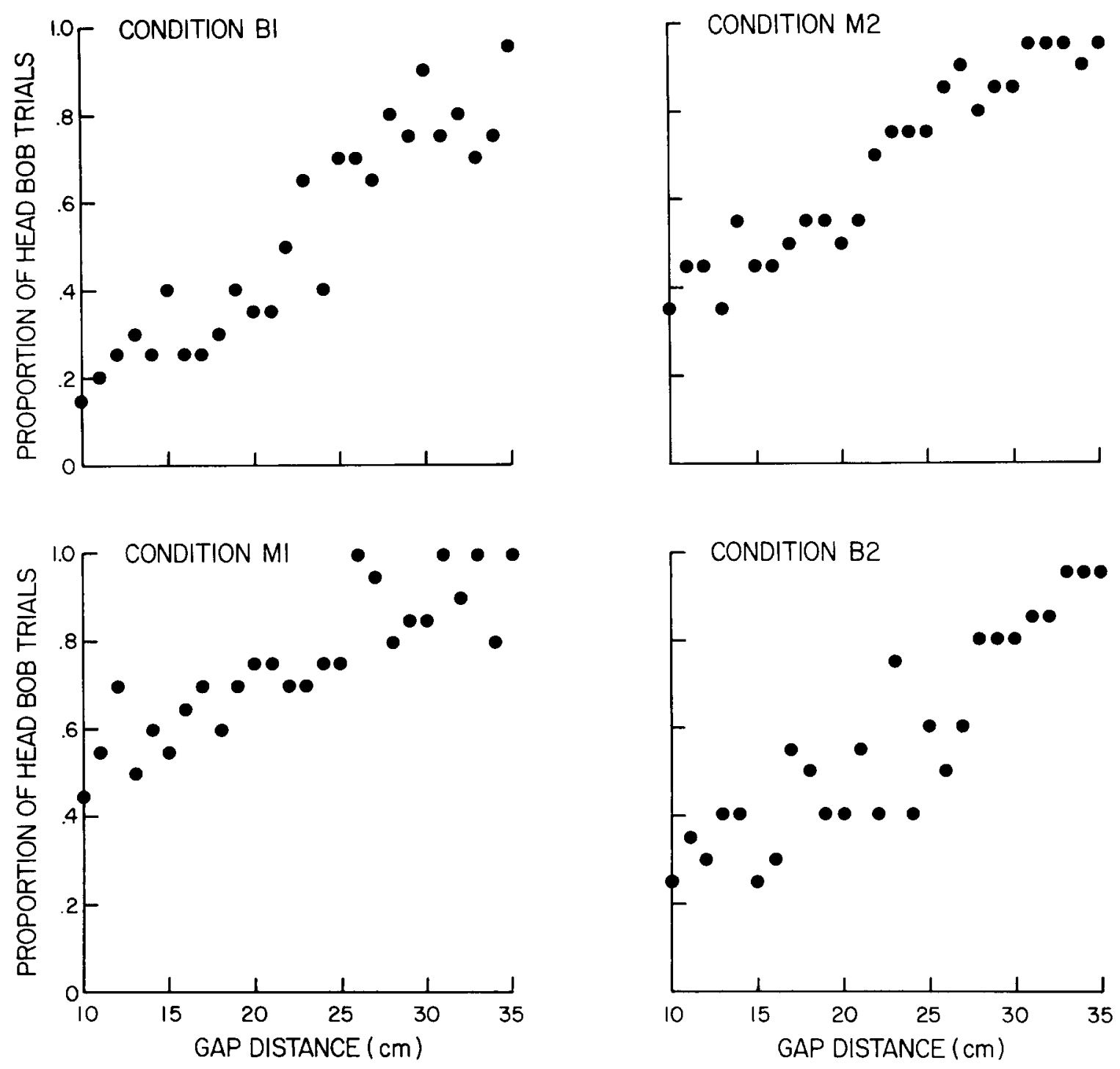

Fig. 4. Plots of the proportion of trials on which at least one head movement was observed as a function of gap distance. Each data point represents a proportion calculated over 20 trials ( 10 animals $\times 2$ trials/animal). Each condition is plotted separately.

bob incidence (HBI) was defined as the proportion of trials on which at least one head bob was observed. This statistic is preferable to a simple count of the mean or total number of head bobs since it is free from the assumption that a longer series of head bobs yields more information than a shorter series. Table I shows the correlation coefficients between landing position variance and head bob incidence calculated over the 10 animals for each session of the 4 different conditions. Two correlation coefficients were calculated: one in which HBI incorporated all observed head bobs, and another in which HBI took account of only those head bobs whose amplitude was equal to or exceeded $1.5 \mathrm{~cm}$. This second calculation eliminated any inflation of HBI scores by small and potentially uninformative head movements. While the correlations between landing position variance and the total number of head bobs were low and the overall coefficient was insignificant $(r=-0.15, \quad \mathrm{df}=56, \quad z=1.12$, $P>0.1$, the correlations with the larger head bobs were stronger and the overall correlation coefficient was significant at the 0.05 level 


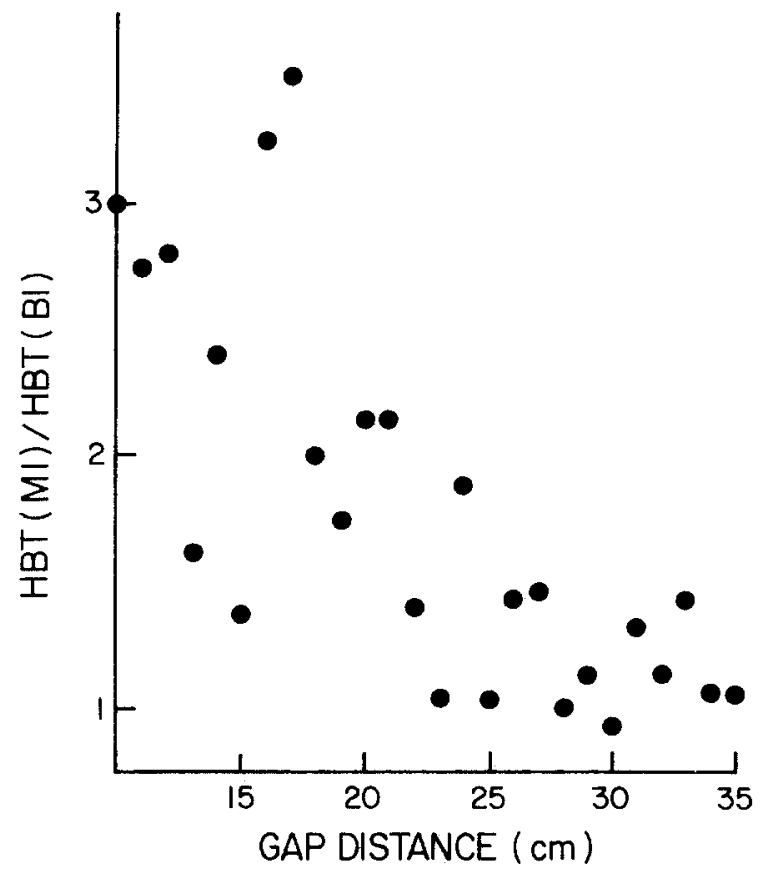

Fig. 5. Each data point on this plot represents the ratio of the number of trials on which at least one head movement was observed in condition Ml to the number observed in condition B1. This ratio is plotted as a function of gap distance.

$(r=-0.33, \mathrm{df}=56, z=2.47, P<0.05)$. In other words, the greater an animal's propensity to produce large head bobs, the more consistent was its landing position across trials.

Head bob incidence was also related to an animal's readiness to initiate a jump. During the

\section{TABLE I}

Correlations of landing position variance with head bob incidence and large amplitude head bob incidence

\begin{tabular}{llcc}
\hline Condition & Session & $\begin{array}{l}\text { All head } \\
\text { bobs }\end{array}$ & $\begin{array}{l}\text { Head bobs } \\
>=1.5 \mathrm{~cm}\end{array}$ \\
\hline B1 & 1 & -0.16 & -0.18 \\
B1 & 2 & -0.01 & -0.43 \\
B2 & 1 & -0.41 & -0.71 \\
B2 & 2 & -0.10 & 0.01 \\
M1 & 1 & -0.22 & -0.49 \\
M1 & 2 & -0.26 & -0.33 \\
M2 & 1 & -0.36 & 0.29 \\
M2 & 2 & -0.46 & -0.33 \\
& & & \\
Overall mean & & -0.15 & $-0.33^{*}$ \\
\hline
\end{tabular}

$* P<0.05$. first few trials of Condition $M 1$, for example, several animals showed an extreme disinclination to jump at any distance at all. This disinclination, quantified as the number of attempts - where the animal positioned itself on the extreme edge of the take-off platform - divided by the number of trials, was strongly related to HBI in the previous binocular sessions (Condition B1). Those animals that had bobbed their heads the most when both eyes were open had the least difficulty in jumping in the monocular condition $\left(r=-0.69, t_{8}=2.7\right.$ $P<0.025$ ).

Since animals were able to jump accurately on many occasions without making any head bobs at all, it was clear that other cues to depth were available. Loom cues produced by forward progression on the take-off platform could have been one such source of information. The size of such forward movements can be calibrated with the change in image size to provide information about distance. To investigate the relationship between potential loom cues and the parallax information derived from head bobbing, the mean forward movement of each animal during the final recorded leg movement was calculated. Since small reflex leg movements sometimes accompany flexion of the neck ${ }^{14}$, only trials that did not contain head bobs were used in calculating this value. In this way, the confounding influence of non-locomotory movements on the calculation of a typical step size for each animal was minimized. Since gerbils typically locomote in saltatory fashion, with rapid forward movements alternating with pauses, the most efficient loom cues are probably those that are generated during a single movement rather than those that are generated across several movements. Furthermore, since the last forward movement before a jump generates the largest change in image size (since this movement brings the animal closest to the target), there is some basis for believing that the amount of horizontal displacement during the last step before a jump provides a reasonable index of the amount of loom information that is available to an animal. Table II shows the correlation coefficients between mean step size and head bob incidence calculated over the 10 animals for each session of the 4 test conditions. In most sessions, 
TABLE II

Correlations between head bob incidence and mean step size

\begin{tabular}{lll}
\hline Condition & Session & $r$ \\
\hline B1 & 1 & -0.71 \\
B1 & 2 & -0.60 \\
B2 & 1 & -0.49 \\
B2 & 2 & -0.58 \\
M1 & 1 & -0.79 \\
M1 & 2 & -0.39 \\
M2 & 1 & 0.14 \\
M2 & 2 & -0.64 \\
Overall mean & & $-0.59^{*}$
\end{tabular}

$* P<0.01$.

mean step size and head bob incidence were inversely related: the larger an animal's step size, the less often it executed head bobs.

In the final experiment that was carried out with 6 of the 10 gerbils, the size of the take-off platform was reduced so that loom cues could not be as easily generated. A proportions analysis of head bob amplitude and velocity as a function of gap size again revealed a significant trend toward larger $\left(\chi^{2}=48.5, P<0.05\right)$ and faster $\left(\chi^{2}=41.5\right.$, $P<0.05)$ head movements with increasing gap distance.

Nevertheless, as Fig. 6 illustrates, a larger number of animals produced head bobs at shorter distances in this situations than they did in the last binocular sessions (Condition B2) of the first experiment. This conclusion is supported by the fact that the ratios of the proportion of animals making head bobs in both situations for each gap size were significantly correlated with the size of the required jump $\left(r=-0.61, t_{7}=2.04\right.$, $P<0.05)$. The mean correlation between landing position variance and head bob incidence was substantially higher $(r=-0.57, \quad \mathrm{df}=12$, $z=1.97, P<0.05)$ than the overall correlation obtained in first study $(r=-0.15, \mathrm{df}=56$, $z=1.12, P>0.1)$.

\section{DISCUSSION}

The results of the present study demonstrate that the Mongolian gerbil is able to make very

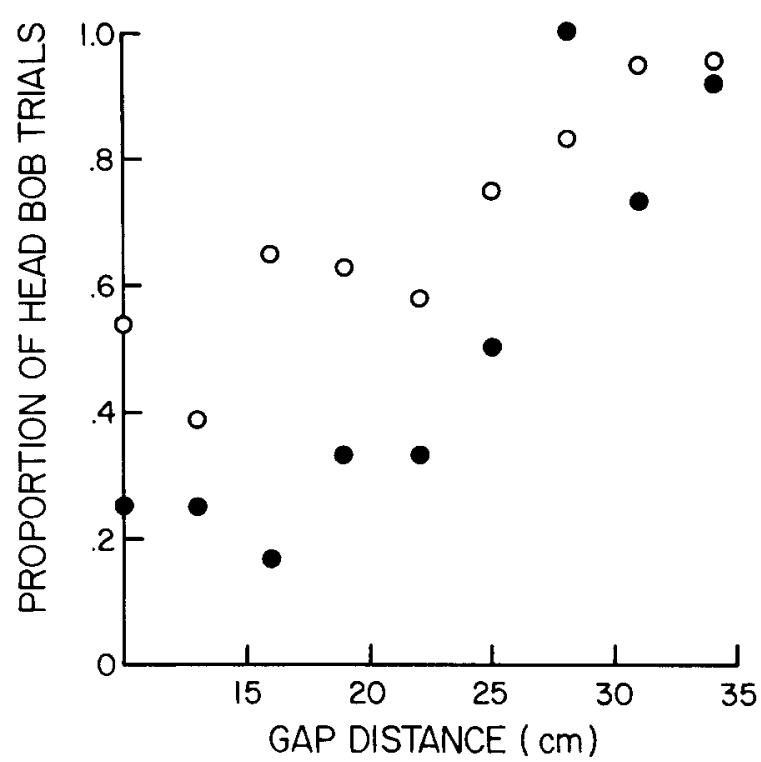

Fig. 6. Plot of the number of trials on which at least one head bob was observed as a function of gap distance. Open circles represent performance of the 6 animals with the short takeoff platform. Filled circles represent their performance during Condition B2.

accurate judgements of how far it must jump to clear a gap in the substrate. The results also suggest that the bobbing movements of the head that often precede the jump are used to generate motion parallax cues for estimating the distance of the required jump.

As the size of the gap which the gerbils were required to jump was increased, the incidence of large amplitude head bobs increased dramatically. The peak velocity of the head bobs also showed a corresponding increase with increasing gap distance. These findings are similar to those reported for the locust ${ }^{2,18}$ and suggest that in order to use translation movements of the head to judge distance, it is necessary to maintain some minimal amount or velocity of retinal image translation. The size or velocity of head bob that would maintain or exceed such a threshold image translation would therefore have to increase with increasing distance to the target object.

It was not possible in these experiments to determine which of the two parameters of image translation, amplitude or velocity, is the more important to preserve while the head is being moved. As with humans ${ }^{9}$, it may be that both play 
a role in providing parallax information. Alternatively, velocity and amplitude may be inseparably linked in such a way that large slow or small fast movements of the head are not possible. The ballistic nature of the head movements, as evidenced by the high correlations between amplitude and peak velocity and between acceleration and peak velocity, lends support to this latter proposal. A similar pattern, in which amplitude is determined by the amount of agonistic muscular activity rather than by the time over which such activity persists, has been found in variety of rapid movements involving the synergistic action of more than one muscle group ${ }^{4,5,7,8}$. It has been suggested that time invariance simplifies the task of coordinating a number of muscle groups which may contribute differentially to a complex movement $^{8}$.

If the head bobs we observed were truly ballistic, they could not have been modified after they were initiated. This might explain why there was such a great deal of heterogeneity in the amplitudes and velocities of head movements at any one gap distance. Indeed, if only large head bobs were produced at long distances, and only small ones at short distances, then this would demand that the amplitude of the head movement be calibrated for a particular gap distance before it was initiated. In other words, the animal would have had to judge the distance of the landing platform before it bobbed its head.

While the relationship between the production of head movements and the accuracy of the jump was quite small, this does not necessarily imply that parallax cues were not important. Head movements may occur for a number of reasons other than for the generation of parallax cues. Small movements of the head often accompany limb movements as a reflexive means of stabilizing an animal's centre of gravity during locomotion ${ }^{14}$. Some head movements might also be expected to occur as the animal simply scans the target area. Unfortunately, in the present study there was no a priori way of distinguishing one kind of head movement from another. However, the fact that the relationship between the incidence of large head movements and accuracy was stronger than that between the incidence of all head movements and accuracy indicates that many of the smaller head movements may not have been involved in the generation of motion parallax information.

No attempt was made in these experiments to limit the number of cues to depth that were available. Retinal disparity, loom, and even static cues from image size could have been used to judge distance. While this makes it difficult to provide quantitative statements about the accuracy of motion parallax, it has made it possible to consider the problem of cue interaction in the determination of depth judgements, an issue that has seldom been broached in the past (but see refs. $3,6)$. The strong correlation between gap distance and head bob incidence shows that the use of motion parallax in this situation was highly distance-dependent. If motion parallax was used more often at longer distances, the implication is that at least one other cue was used at shorter distances but that the efficacy of these other cues diminished rapidly as the distance of the landing platform increased. Furthermore, the increase in vertical head movements at short distances during the first monocular condition suggests that these other cues might have been dependent upon the use of both eyes.

Any change in performance that follows the closing of an eye is usually attributed to a loss of retinal disparity cues. It is possible that such cues were used in the present experiment. It is interesting in this regard that the head movements observed in these experiments were all vertical movements. In humans, psychophysical data indicate a close relationship between the systems involved in stereopsis and in motion parallax ${ }^{15}$. Since, in the present case, one system uses differences in vertical retinal position and the other uses horizontal disparities, it would be surprising if the same close relationship were to be found.

The data suggest that at least some of the changes observed in the monocular conditions were the result of loss of information from loom, the change in image size brought about by an animal's own forward movement. In humans, channels that are selective for approaching objects can be stimulated monocularly ${ }^{13}$. The presence of interocular transfer of loom aftereffects, however, indicates binocular interaction of loom 
detector channels, probably at the cortical level. While loom cues are potentially useful in a monocular situation then, it is possible that the closure of one eye might have some effect, perhaps only a transient one, on the efficacy of loom, particularly in an animal that initially learned to perform a depth judgement with both eyes open. This the pattern of performance was observed in the present experiment.

The purported use of loom cues is supported by a number of other observations. The negative correlation between the mean size of the final step and an animal's head bob incidence suggests that those animals that had the least opportunity to use loom to judge depth were the most likely to make head movements to generate parallax cues. These animals were also the ones that were the least disturbed by having one eye closed in Condition $\mathrm{M} 1$, presumably because they were the least dependent on information provided by loom. Additional support for the hypothesis that loom was the second cue used by the animals in this task comes from the results of the experiment that used a short take-off platform. In this situation, the incidence of head bobs was raised at short distances in a manner similar to that observed in the first monocular condition. This result would be expected if the animals' opportunity to generate loom cues was reduced.

In summary, the findings reported here constitute the strongest evidence to date for the use of motion parallax cues in a mammal other than man. In its natural environment, an organism seldom encounters the optimal conditions for the use of any one depth cue. Instead, the animal must combine a series of estimates from a number of sources and weight those estimates according to the sensitivity of these sources in the prevailing conditions. The discovery of a system where at least two depth cues are used with regularity provides the opportunity to further investigate the psychophysics and neural structures that underly such cue interactions.

\section{ACKNOWLEDGEMENTS}

This work was supported by Grant A6313 from the Natural Sciences and Engineering Research Council of Canada to M.A.G.

\section{REFERENCES}

1 Baker, A.G. and Emerson, V.F., Grating acuity of the Mongolian gerbil, Behav. Brain Res., 8 (1983) 195-209.

2 Collett, T., Peering: a locust behavior pattern for obtaining motion parallax information, J. exp. Biol., 76 (1978) 237-241.

3 Collett, T. and Harkness, L., Depth vision in animals. In D.J. Ingle, M.A. Goodale and R.J.W. Mansfield (Eds.), The Analysis of Visual Behavior, MIT Press, Cambridge, MA, 1981, pp. 111-176.

4 Cooke, J.D., The organization of simple, skilled movements. In G.E. Stelmach and J. Racquin (Eds.), Tutorials in Motor Behavior, Elsevier, Amsterdam, 1980 , pp. 199-212.

5 Fisk, J.D. and Goodale, M.A., The relationship between eye and limb movements during visually guided reaching, submitted.

6 Freeman, R.B., Theory of cues and the psychophysics of visual space perception, Psychon. Monogr., Suppl. 3, No. 13, Whole No. 45 (1970) 171-181.

7 Freund, H.-J. and Budingen, H.J., The relationship between speed and amplitude of the fastest voluntary contractions of human arm muscles, Exp. Brain Res., 13 (1978) $1-12$.

8 Hallet, M. and Marsden, C.D., Ballistic flexion movements of the human thumb, J. Physiol. (Lond.), 294 (1979) 33-50.

9 Hell, W., Movement parallax: an asymptotic function of amplitude and velocity of head motion, Vision Res., 18 (1978) 629-635.

10 Lashley, K.S. and Russell, J.T., The mechanism of vision. XI. A preliminary test of innate organization, J. genet. Psych., 45 (1934) 136-144.

11 Mlinar E.J. and Goodale, M.A., Cortical and tectal control of visual orientation in the gerbil: evidence for parallel channels, Exp. Brain Res., 55 (1984) 33-49.

12 Olson, C.R., Spatial localization in cats reared with strabismus, J. Neurophys., 43 (1980) 792-806.

13 Regan, D. and Beverley, K.I., Looming detectors in the human visual pathway, Vision Res., 8 (1978) 415-421.

14 Roberts, T.D.M., Neurophysiology of Postural Mechanisms, Butterworth, London, 1967.

15 Rogers, B. and Graham, M., Similarities between motion parallax and stereopsis in human depth perception, Vision Res., 22 (1982) 261-270.

16 Russell, J.T., Depth discrimination in the rat, J. genet. Psychol., 40 (1931) 136-159.

17 Walk, R.D. and Gibson, E.J., A comparative and analytical study of visual depth perception, Psychol. Monogr., 75 (1961) 519 .

18 Wallace, G.K., Visual scanning in the desert locust Schistocerca gregaria (Forskal), J. exp. Biol., 36 (1959) 512-525. 